\title{
Therapeutic Strategies for Clinical Trials Targeting Renal Recovery
}

\author{
Kathleen D. Liu \\ Divisions of Nephrology and Critical Care Medicine, Departments of Medicine and Anesthesia, University of \\ California, San Francisco, Calif., USA
}

\section{Key Words}

Acute kidney injury · Acute respiratory distress syndrome · Best care practices $\cdot$ Renal recovery

\begin{abstract}
Although at present we have developed consensus definitions for acute kidney injury (AKI) and there is a major focus on the treatment and prevention of $\mathrm{AKI}$ in high-risk populations, little is known about renal recovery per se and the impact of therapies on renal recovery. Here, we will focus on the specifics of clinical trial design relevant to studies of AKI recovery. While certain design considerations will be common to all trials, others elements will be more specific for studies focused on best practice or novel therapeutics. We will first discuss clinical trial considerations for all studies followed by specific proposals for best practice trials and therapy trials. Other presentations during this round table have focused on other aspects that are highly important for clinical trial design, including definitions for recovery, the potential use of biomarkers, as well as clinical trial endpoints, so we will not address these issues here.

(C) 2014 S. Karger AG, Basel
\end{abstract}

Targeting Recovery from Acute Kidney Injury: Round Table Conference at the 19th International Conference on Continuous Renal Replacement Therapies (Manchester Grand Hyatt, San Diego, Calif., USA, March 2-3, 2014).

\section{KARGER}

E-Mail karger@karger.com www.karger.com/nec
Apart from the selection of therapy itself, there are many important and practical clinical trial design issues relevant to studies of acute kidney disease (AKI) recovery. For completeness sake, we should note at the outset that there are unmodifiable risk factors for adverse outcomes of AKI (e.g., lack of renal recovery) that cannot be modified by treatment. These include age, gender, race/ethnicity, as well as comorbidities that are unmodifiable in the short term, such as obesity. In the same category of comorbidities, the level of premorbid kidney function and renal reserve may also be considered. That is, there are likely individuals for any given therapeutic who have such advanced chronic kidney disease and/or so little renal reserve that they are unlikely to benefit from a novel therapy. However, the level of premorbid kidney function and renal reserve are also critical aspects of patient selection since patients may be at higher risk for poorer renal recovery based on these factors.

In terms of practical clinical trial design, the first aspect to consider is the population of interest. Here, a major consideration is who has the potential to benefit from the treatment of interest? This likely relates both to the level of estimated glomerular filtration rate as well as renal reserve. For example, it is clear from prior studies of dialysis-requiring AKI that patients with baseline normal renal function have the highest inpatient mortality rates yet have the highest rate of renal recovery (defined as dialysis discontinuation) among survivors [1]. In contrast, 
studies of patients with advanced chronic kidney disease have the lowest rates of inpatient mortality, yet relatively few of these patients experience renal recovery $[2,3]$. With regard to renal reserve, at present, we have no measures of renal reserve that are used in widespread clinical practice. However, the clinical importance of renal reserve is apparent: 2 patients with similar levels of estimated glomerular filtration rate may have very different levels of renal reserve and consequently have different potential for recovery after AKI [4]. Consequently, studies of novel therapies for recovery need to consider what group of patients may have enough reserve as well as underlying kidney function to benefit from therapy. This may depend in part on the proposed mechanism of action of a novel therapeutic. Along the same lines, clinical trial design must consider the number of patients anticipated to experience spontaneous recovery in the underlying study population, because this variable will impact the number of patients needed to treat to see benefit.

The next design consideration is when to treat patients with a novel therapy for renal recovery. One potential time point for treatment is as close to the injury as possible. Here, the hypothesis is that modifying the course of AKI may modify recovery. Specifically, we know from prior studies $[5,6]$ that patients who have more episodes of AKI or more severe episodes of AKI are more likely to experience de novo chronic kidney disease or chronic kidney disease progression. For ongoing and future studies of novel therapies for the treatment of AKI, this may mean considering extending follow-up time beyond the index hospitalization. Although such follow-up can be logistically challenging, it is cost-effective when considering the overall costs of such trials. Another potential time point is to treat patients after some measure of disease severity, for example, after patients start dialysis. This has the benefit of studying patients who have a similar severity of illness. Importantly, the time point for treatment is likely highly dependent on the nature of the proposed therapy itself and its proposed mechanism of action.

In terms of what therapies to consider, it is useful to consider conceptual models proposed for renal recovery [7]. It has been proposed that chronic kidney disease occurs as a result of failed differentiation and subsequent tubular atrophy after AKI. Either best supportive care or novel therapeutics currently in development may promote normal regeneration after AKI. For example, we have compelling data from prior studies of the acute respiratory distress syndrome (ARDS) that suggest best supportive care practices including low tidal volume ventilation have substantially reduced patient mortality over the past 15-20 years. In the first large ARDS Network clinical trial of low versus high tidal volumes, the mortality rate was $39.8 \%$ in the patients ventilated with high tidal volumes [8]. In the most recent ARDS Network clinical trial, the overall mortality rate was $22.7 \%$ [9]. This is despite the fact that over that same 15-year period, severity of illness in this patient population has increased; the mean APACHE (Acute Physiology and Chronic Health Evaluation) III score was 82 in the first trial and has increased to 91 . This decrease in mortality is probably due to the use of low tidal volume ventilation as a standard therapy, as well as other best care practices that have been standardized, for example time to antibiotic therapy in patients with sepsis.

In terms of AKI, what best practices could be tested? First, we could consider the use of renal replacement therapy (RRT). In a companion paper, Schneider and Bagshaw [this issue] have considered the impact of a number of aspects of RRT on renal recovery, including the use of any versus no RRT, the timing of RRT, RRT modality, the selection of the dialysis membrane, and the selection of anticoagulation (specifically, the use of citrate anticoagulation). There has been much interest in the impact of RRT modality on renal recovery given the experimental observation that hypotension after AKI is associated with prolonged renal recovery. A large meta-analysis by Schneider et al. [10] suggested a benefit for continuous RRT over intermittent RRT for renal recovery. However, this meta-analysis is a composite of both observational studies and randomized clinical trial data, with the randomized trial data overall more neutral than the observational data. Furthermore, a large retrospective study of patients hospitalized at a single center suggested no benefit to renal recovery with continuous RRT and was not included in this meta-analysis [Liang and Kellum, pers. commun.]. Overall, it seems reasonable to include renal recovery and long-term outcomes in studies of RRT and in particular randomized clinical trials. However, the RRT population presents truly just the 'tip of the iceberg' of AKI and testing the impact of RRT on recovery will only be informative for a small proportion of the AKI population.

Furthermore, one of the challenges of these studies is understanding whether or not it is RRT itself or processes associated with RRT that matter for renal recovery. For example, one of the mechanisms that has been proposed for the impact of RRT modality on recovery is the association of intermittent RRT with hypotension, which may delay renal recovery. In the landmark Acute Renal Failure Trial Network Study, approximately 17\% of intermittent 
dialysis treatments were complicated by hypotension [11]. Consequently, patients receiving intermittent RRT may experience more episodes of hypotension and this may be associated with delayed renal recovery, rather than the modality itself. Along the same lines, little is known about the impact of blood pressure control after AKI on recovery despite a number of animal studies suggesting that hypertension may occur after ischemia-reperfusion injury in animal models. However, a challenge to studying blood pressure control after AKI includes the fact that effect size may be small, and a very large study may be required. Thus, at present, best supportive care targets for blood pressure after AKI are unknown.

Another best practice to consider is fluid overload management. A number of studies have suggested that fluid overload is associated with adverse outcomes, including lack of renal recovery at 1 year [12]. Again, whether or not this is due to physiological effects of the fluid or due to the association of fluid with other diseases associated with poor outcomes, such as sepsis, is unknown at this time.

The impact of nutrition on AKI recovery is also unknown. Animal studies have suggested that caloric restriction after AKI is associated with a decreased rate of glomerulosclerosis and tubular atrophy [7]. However, in the human setting, getting sufficient calories and protein appears to be more of a challenge than administering too many calories. Furthermore, in other forms of criticalness, antioxidant and anti-inflammatory supplements have not been shown to be of benefit. Nonetheless, this is an important consideration since nutrition is provided to all patients in the hospital, and interventions here may not be expensive in and of themselves.

A commonsense best practice approach to promote renal recovery consists of early recognition of AKI, workup for reversible causes, avoidance of nephrotoxins, and close follow-up of patients with in-hospital AKI after discharge. Questions here remain about the intensity of follow-up and by whom follow-up should be performed for the most cost-effective approach. Finally, with regard to best practices, there are a number of risk factors for adverse short-term outcomes (e.g., mortality) from AKI where the association with long-term outcomes, including renal recovery, is unknown. These include oliguria, anemia, transfusion, and the timing of antimicrobial therapy. These could potentially be the subject of future studies as well.

Turning to potential novel therapies, presentations during this round table focused on a number biological pathways involved in renal recovery. Here, one of the

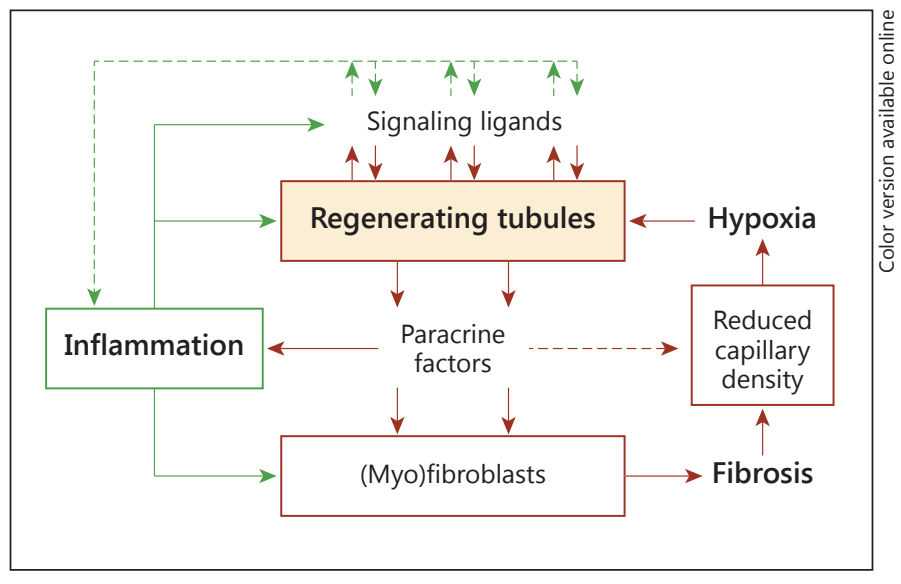

Fig. 1. Potential mechanisms leading to inflammation and fibrosis after AKI. Reproduced from Venkatachalam et al. [7].

challenges is to try to identify novel targets that may be modulated by existing molecules that have already undergone preclinical and clinical development. The potential 'repackaging' of approved agents for renal recovery is an attractive pathway for drug development as it may require less funds than identifying a novel compound from scratch and taking it fully through the clinical development pipeline (though still likely to be more expensive than a study of best practices). These novel therapies could impact a number of potential targets as shown in figure 1.

An agent that may affect biological pathways reviewed during this round table is formoterol, a long-acting $\beta$-agonist approved for the treatment of asthma and chronic obstructive pulmonary disease. This molecule decreases ischemia-reperfusion injury in animal models and leads to accelerated renal recovery. Recently, Jesinkey et al. [13] suggested that the mechanism of action for formoterol in renal recovery is to improve mitochondrial function and decrease mitochondrial injury during AKI.

During this round table, Zager et al. [14] reviewed the use of atrasentan, a molecule that has gone through substantial preclinical development for the treatment of prostate cancer. In an animal model of ischemia-reperfusion injury, atrasentan had no impact on AKI, which was defined by short-term blood urea nitrogen and creatinine measurements. However, over a longer period atrasentan was associated with decreased renal fibrosis and atrophy. Further studies are needed to understand the impact of atrasentan on renal function at these longer time points. Nonetheless, atrasentan represents a compelling target for future studies since the molecule has been studied in humans previously. 
An appealing third class of novel therapeutics for AKI is cell-based therapy. Dr. Humphreys reviewed at length the potential role of mesenchymal stem cells in renal recovery at this round table conference. This includes their well-described anti-inflammatory effects as well as their effects on a number of endothelial and epithelial cell signaling pathways. In other models of epithelial injury, it has recently been shown that mesenchymal stem cells may directly transfer mitochondria to the injured epithelial cells via connexin- 43 to restore normal cellular bioenergetics [15]. These cells have been used in over 2,000 humans for a variety of disease indications including graft-versus-host disease, Crohn's disease, acute myocardial infarction, and ARDS. There are ongoing phase 2 trials (AlloCure) to test whether or not these cells can prevent AKI after high-risk cardiopulmonary bypass surgery. With regard to their use as a novel therapy for renal recovery, the fact that these cells have multiple mechanisms of action makes them an attractive therapeutic agent. Potential challenges to the development of mesenchymal stem cell therapy include the fact that this therapy is relatively expensive and requires coordination with the bone marrow transplantation laboratory for administration. Advantages of this therapy include the fact that it appears to be well tolerated and has multiple mechanisms of potential action.

Finally, other molecules with potential novel mechanisms of therapy to consider include resveratrol, which may affect both apoptosis and hypoxia-inducible factor-1 signaling after AKI, and citrus pectins, which may decrease galectin expression and attenuate renal fibrosis after AKI. Again, studies of novel therapeutics should strongly favor agents that already have strong preclinical and clinical development programs.

In summary, this is an exciting time in the AKI world. We now have a better understanding of the long-term impact of AKI on chronic kidney disease and other patient outcomes, including mortality. Consequently, a major focus of the field has shifted to the impact of therapy on renal recovery. We have outlined here a number of aspects of best clinical practices as well as novel therapeutics that may be amenable to study in clinical trials to promote AKI recovery.

\section{References}

1 Schiffl H, Lang S, Fischer R: Daily hemodialysis and the outcome of acute renal failure. $\mathrm{N}$ Engl J Med 2002;346:305-310.

$>2$ Lo LJ, Go AS, Chertow GM, McCulloch CE, Fan D, Ordonez JD, Hsu CY: Dialysis-requiring acute renal failure increases the risk of progressive chronic kidney disease. Kidney Int 2009;76:893-899.

$\checkmark 3$ Hsu CY, Ordonez JD, Chertow GM, Fan D, McCulloch CE, Go AS: The risk of acute renal failure in patients with chronic kidney disease. Kidney Int 2008;74:101-107.

$\checkmark 4$ Bosch JP, Saccaggi A, Lauer A, Ronco C, Belledonne M, Glabman S: Renal functional reserve in humans. Effect of protein intake on glomerular filtration rate. Am J Med 1983;75: 943-950.

5 Chawla LS, Amdur RL, Amodeo S, Kimmel PL, Palant CE: The severity of acute kidney injury predicts progression to chronic kidney disease. Kidney Int 2011;79:1361-1369.

$\checkmark 6$ Thakar CV, Christianson A, Himmelfarb J, Leonard AC: Acute kidney injury episodes and chronic kidney disease risk in diabetes mellitus. Clin J Am Soc Nephrol 2011;6:25672572 .
7 Venkatachalam MA, Griffin KA, Lan R, Geng H, Saikumar P, Bidani AK: Acute kidney injury: a springboard for progression in chronic kidney disease. Am J Physiol Renal Physiol 2010;298:F1078-F1094.

$>8$ The ARDS Network: Ventilation with lower tidal volumes as compared with traditional tidal volumes for acute lung injury and the acute respiratory distress syndrome. $\mathrm{N}$ Engl J Med 2000;342:1301-1308.

$\checkmark 9$ Rice TW, Wheeler AP, Thompson BT, Steingrub J, Hite RD, Moss M, Morris A, Dong N, Rock P: Initial trophic vs full enteral feeding in patients with acute lung injury: the EDEN randomized trial. JAMA 2012;307:795-803.

-10 Schneider AG, Bellomo R, Bagshaw SM, Glassford NJ, Lo S, Jun M, Cass A, Gallagher $\mathrm{M}$ : Choice of renal replacement therapy modality and dialysis dependence after acute kidney injury: a systematic review and metaanalysis. Intensive Care Med 2013;39:987997.

11 Palevsky PM, Zhang JH, O’Connor TZ, Chertow GM, Crowley ST, Choudhury D, Finkel K, Kellum JA, Paganini E, Schein RM, Smith MW, Swanson KM, Thompson BT, Vijayan A, Watnick S, Star RA, Peduzzi P: Intensity of renal support in critically ill patients with acute kidney injury. N Engl J Med 2008;359:7-20.
12 Heung M, Wolfgram DF, Kommareddi M, $\mathrm{Hu}$ Y, Song PX, Ojo AO: Fluid overload at initiation of renal replacement therapy is associated with lack of renal recovery in patients with acute kidney injury. Nephrol Dial Transplant 2012;27:956-961.

13 Jesinkey SR, Funk JA, Stallons LJ, Wills LP, Megyesi JK, Beeson CC, Schnellmann RG: Formoterol restores mitochondrial and renal function after ischemia-reperfusion injury. J Am Soc Nephrol 2014;25:1157-1162.

14 Zager RA, Johnson AC, Andress D, Becker K: Progressive endothelin-1 gene activation initiates chronic/end-stage renal disease following experimental ischemic/reperfusion injury. Kidney Int 2013;84:703-712.

15 Islam MN, Das SR, Emin MT, Wei M, Sun L, Westphalen K, Rowlands DJ, Quadri SK, Bhattacharya S, Bhattacharya J: Mitochondrial transfer from bone-marrow-derived stromal cells to pulmonary alveoli protects against acute lung injury. Nat Med 2012;18:759-765. 